\title{
Camada-limite em placa plana: revisitando o Método da Similaridade
}

\author{
Flate plate boundary layer. revisiting the Similarity Method
}

\author{
1 Élcio Nogueira elcionogueira@hotmail.com \\ 2 Marcus Vinicius Ferreira Soares \\ 1 Professor Assistente Dr. do UniFOA, Professor Dr. da AEDB e Professor Adjunto do Departamento de Mecânica e Energia - DEM
- FAT/UERJ - Resende.
2 Aluno do Programa de Mestrado em Meteorologia na UFRJ
}

\section{Resumo}

Ao escrever o presente trabalho, tem-se em mente os estudantes que se iniciam em Mecânica dos Fluidos e se deparam com o conceito de camada-limite. Nesse sentido, como primeira abordagem, introduz-se a solução de Blasius para placa plana, uma vez que se trata de um problema básico em camada-limite e possibilita ao estudante antever o grau de dificuldade a ser enfrentado em problemas mais complexos. Além disso, acredita-se que seja um assunto a ser apresentado e discutido em consonância ao estudo introdutório das Equações de Navier-Stokes, uma vez que se trata de primeira aproximação consolidada, historicamente falando, desse conjunto de equações. 0 foco principal do trabalho reside no Método da Similaridade, uma vez que Blasius o utilizou para obtenção da solução da camada-limite hidrodinâmica em regime laminar, resultados estes que foram de grande impacto na moderna Mecânica dos Fluidos. Não se limita à solução do Problema de Blasius, mas estende-se a análise para o problema da solução da Equação da Energia, ou seja, na obtenção da camada-limite térmica, onde pode-se obter o perfil de temperatura e o gradiente de temperatura, ao longo da camada-limite. Introduz-se o Método Integral da Quantidade de Movimento, uma vez que equação integral da quantidade de movimento possibilita determinar, com razoável precisão, a espessura da camada-limite turbulenta. Apresentam-se soluções para regime turbulento, em comparação ao regime laminar, tanto para perfil de velocidade quanto para perfil de temperatura. Os conceitos e procedimentos podem servir de base para soluções de camada-limite que não satisfazem o conceito de similaridade, como para problemas de camada-limite em geral.

\section{Palavras-chave}

Camada-limite em placa plana. Método da Similaridade. Método Integral. Método de Runge Kutta. Camada-limite hidrodinâmica. Camada-limite térmica.

\begin{abstract}
In writing the present work it was considered the students who start in fluid mechanics and meet the concept of boundary layer. In this sense, as a first approach, the Blasius solution for flat plate is introduced, since it is a basic problem in the boundary layer and allows the student to understand the degree of difficulty to be faced in more complex problems. In addition, it is considered to be a subject to be presented and discussed in line with the introductory study of the Navier-Stokes equations, since it is the first consolidated approach, historically speaking, of this set of equations. The focus lies in the method of similarity, since Blasius used it to obtain the solution of the hydrodynamic boundary layer in laminar regime, and the results were of great impact in modern fluid mechanics. The work is not limited to the solution of the Blasius problem, but extend the analysis to the problem of solution of the energy equation, that is, in obtaining the thermal boundary layer, where one can get the temperature profile and the temperature gradient along the boundary layer. The integral method is used, since the integral equation of the momentum determines, with reasonable accuracy, the thickness of the turbulent boundary layer. Solutions for turbulent regime compared to the laminar regime, both for velocity profile and for temperature profile. The concepts and procedures can serve as a basis for boundary layer solutions that do not satisfy the concept of similarity, and for general boundary layer problems.
\end{abstract}

\section{Keywords}

Flat plate boundary layer; Similarity method; Integral method; Runge Kutta method; Hydrodynamic boundary layer; Thermal boundary layer

\section{Como você deve citar?}

NOGUEIRA, Élcio; SOARES, Marcus Vinicius Ferreira. Camada-limite em placa plana: revisitando o Método da Similaridade. Cadernos UniFOA, Volta Redonda, n. 37, p. 15-31, ago. 2018. 


\section{INTRODUÇÃO}

Durante o século XIX, William Froude introduziu o conceito denominado "Lei da Similaridade", associado às suas pesquisas em dinâmica dos fluidos. A lei da similaridade, nesse caso, se aplicava às ondas em superfícies livres. Ao final desse mesmo século, Osborne Reynods formula uma lei para escoamento viscoso, ao mesmo tempo em que executa o seu famoso experimento no qual se demonstra a transição de escoamento em regime laminar para regime turbulento, em dutos de seção reta circular. Ainda, Saint-Vernant, Navier e Stokes generalizam as equações de Euler para escoamento não viscoso, abrindo novas perspectivas de pesquisas nas áreas teóricas e aplicadas em fluidodinâmica. É fato notório que, no final do século XIX, havia uma grande discrepância entre os resultados da hidráulica (hidrodinâmica prática) e os resultados oriundos dos modelos teóricos da fluidodinâmica, baseados na teoria do escoamento potencial (escoamentos ideais). A generalização dos modelos teóricos, com a introdução das denominadas Equações de Navier-Stokes, era um motivo para grande euforia e ótimas perspectivas. Entretanto, em função da complexidade dos sistemas de equações, o avanço na solução das Equações de Navier-Stokes permaneceu estagnado. De fato, apesar de todo avanço teórico, envolvendo formulações matemáticas de soluções de sistemas de equações diferenciais parciais, as dificuldades momentâneas para obtenção de soluções, mesmo para as situações mais simples, eram intransponíveis. Tornava-se evidente a necessidade de novas hipóteses e conceitos, no intuito de obter simplificações que possibilitassem a solução de tais equações e a realização de comparações com dados experimentais.

Logo no início do século XX, 1904, Ludwig Prandtl postula que o escoamento em torno de um corpo liso deveria ser dividido em duas regiões: 1 - Próximo à superfície a viscosidade governa 0 escoamento; 2 - Distante da superfície a viscosidade tem efeito desprezível, e o escoamento satisfaz a teoria de Euler. Evidentemente, nesse caso, a solução deve satisfazer as condições apropriadas na fronteira dessas duas regiões. Tais hipóteses, e a equações derivadas a partir das equações de NavierStokes, receberam o nome de "Formulação em Camada-limite".

O conceito de camada-limite (CL), atribuído a Prandtl, abriu o caminho para soluções aproximadas de problemas práticos importantes na área de engenharia.

O aluno de doutorado, orientado por Prandtl, Heinrich Blasius, utilizou os conceitos de camada-limite e lei da similaridade para obtenção da solução da camada-limite em regime laminar, em placa plana (1908).

Como formulado por Prandtl, em 1904, as equações de Navier-Stokes simplificadas, para camada-limite em placa plana são Evans (1968); Kays and Crawford (1983); Schlichting(1968); Shames (1973):

$$
\begin{aligned}
& u\left(\frac{\partial u}{\partial x}\right)+v\left(\frac{\partial u}{\partial y}\right)=\vartheta\left(\frac{\partial^{2} u}{\partial y^{2}}\right) \\
& \frac{\partial u}{\partial x}+\frac{\partial v}{\partial y}=0
\end{aligned}
$$

sujeitas às condições de contorno:

$$
u(y=0)=v(y=0)=0 \text { e } u(y \rightarrow 0)=U
$$


onde u e v são as componentes do campo de velocidade nas direções x e y, respectivamente; $\vartheta$ é a viscosidade cinemática e $U$ é a velocidade da corrente livre (também denominada de região não perturbada).

A técnica matemática associada ao Método da Similaridade é, algumas vezes, denominada técnica da combinação de variáveis, aplicável a certos problemas onde o comprimento característico é determinado por taxa de variação, e menos que por considerações geométricas. Tais problemas geralmente envolvem regiões semi-infinitas, e podem ser lineares ou não lineares. Para camada-limite estacionária, o fator de escala é a espessura da camada-limite, a qual é tipicamente uma posição que varia ao longo da superfície. A solução por similaridade existe, se a equação de transporte e suas condições de contorno permitirem uma transformação homogênea. Isso implica em encontrar uma relação entre as variáveis independentes, no caso $x$ e $y$, para apenas uma variável, $\eta$, que seria uma combinação entre y e uma função de $x$, a ser determinada. 0 primeiro passo na busca de uma transformação por similaridade é expressar a variável dependente na forma de um produto de funções, sendo que uma delas envolve o parâmetro de similaridade, ou seja:

$$
u(x, y)=U \cdot f^{\prime}(\eta) \quad ; \quad \eta=\eta(\mathrm{y}, \mathrm{h}(\mathrm{x}))
$$

Se for possível uma tal transformação, então o perfil de velocidade tem a propriedade de ser proporcional em diferentes posições de $x$, através de uma transformação de varáveis $h(x)$ e $Y$, que define que $\eta[h(x)$ é o fator de proporcionalidade para a coordenada $y$ ], e a velocidade externa $U(x)$ [fator de proporcionalidade para a velocidade u].

Ao se expressar $u(x, y)$, tem-se como condição de contorno:

$$
\left(\frac{u}{U}\right)_{y=0}=\left[f^{\prime}(\eta)\right]_{y=0}=0
$$

- Condição de contorno de não deslizamento;

e

$$
\lim _{y \rightarrow \infty}\left(\frac{u}{U}\right)=\lim _{y \rightarrow \infty} f^{\prime}(\eta)=1
$$

A velocidade dentro da CL tende assintoticamente para o escoamento externo.

Blasius, em 1907, reduziu o sistema de equações parciais acima, Equações 01 - 03, em uma equação diferencial ordinária de terceira ordem, com as definições de novas variáveis de similaridade:

$$
\eta=\left(\frac{y}{2}\right) \cdot\left(\frac{U}{v x}\right)^{1 / 2} \quad e \quad \psi=\left(\frac{U x}{v}\right)^{\frac{1}{2}} \cdot f
$$

o que resulta na equação (EVANS, 1968; SHAMES, 1973):

$$
f^{\prime \prime \prime}+f \cdot f^{\prime \prime}=0
$$


com as seguintes condições de contorno:

$$
\begin{array}{ll}
\eta=0, & f=f^{\prime}=0 \\
\eta \rightarrow \infty, & f^{\prime} \rightarrow 1.0
\end{array}
$$

Blasius resolve o problema de valor de contorno acima, através de uma expansão em série, ao invés de tentar uma solução direta. Após testes, em uma variedade de situações, a solução de Blasius foi capaz de demonstrar a importância e o poder do conceito de camada-limite, conforme formulado por Prandtl (HAGER, 2003). De fato, apenas para citar um exemplo, como solução direta da formulação de Blasius, a força $F$, que atua em uma placa plana de largura $B$ e comprimento $L$, é obtida por:

$$
F=f_{0}{ }^{\prime \prime} \cdot \rho \cdot B \cdot\left(\vartheta \cdot L \cdot U^{3}\right)^{1 / 2}
$$

onde $\rho$ é a densidade do fluido.

Os resultados relacionados com camada-limite foram de grande impacto na moderna Mecânica dos Fluidos, e o Método da Similaridade continua sendo aplicado para obtenção de soluções em camada-limite, regime laminar ou turbulento (MYERS, 2010; PUTTKAMMER, 2013; BOGNAR, 2011; BHATTACHARYYA, 2016; RAHMAN, 2011; ABBASI, 2014; MARTIN, 2006; CASTILHO, 1997).

Um método numérico, de grande precisão para solução de camada-limite, é a rotina de Runge Kutta de $4^{\mathrm{a}}$ ordem, porque, apesar de relativamente simples, o problema não comporta solução analítica. A rotina de Runge Kutta é um processo em marcha, que resolve problema de condição de contorno, integrando a equação a partir de uma extremidade, onde as condições são conhecidas até a outra extremidade. Entretanto, em camada limite não se conhece o valor de $f^{\prime \prime}(0)$ e $\operatorname{sim} f^{\prime}((O)=1$, para se dar início ao processo de integração. A dificuldade é superada, atribuindo-se um valor para f"(0), o que pode ser obtido de forma aproximada pelo Método de Série de Potência, diminuindo o tempo de processamento (BUTCHER, 2016). Esse procedimento, associado ao denominado "Shooting Method" (ODERINU, 2014), no qual pode se aplicar rotinas do tipo bissecção ou Newton-Raphson, possibilita a solução com precisão definida. Entretanto, como advertência aos que pretendem implementar o procedimento, é importante sentir a sensibilidade do mesmo, para que se possa definir o valor associado ao infinito.

O Método da Similaridade pode ser aplicado para camada-limite turbulenta, desde que o escoamento esteja em equilíbrio, como demonstrado por Luciano Castilho (1997). De fato, muitos escoamentos podem ser classificados como escoamento em equilíbrio, contrário ao conceito tradicional que estipula que escoamentos em equilíbrio são difíceis de encontrar na prática. 0 escoamento em placa plana, com gradiente de pressão nulo, ou não, regime laminar ou turbulento, é um escoamento em equilíbrio, conforme os critérios definidos por Luciano Castilho.

Como demonstrado por Evans (1968), a equação final da energia para camada-limite similar, é dada por:

$$
\theta^{\prime \prime}+\operatorname{Prf} \theta^{\prime}=0
$$


onde as derivadas dizem respeito à variável $\eta$. As condições de contorno, às quais a variável $\theta$ (temperatura adimensional) deve satisfazer, são:

$$
\begin{aligned}
& \eta=0, \quad \theta=0 \\
& \eta \rightarrow \infty, \quad \theta \rightarrow 1
\end{aligned}
$$

A última condição acima significa que a temperatura tende para a temperatura da corrente livre, para valores de $\eta$ significativamente elevados. De acordo com a Equação 08 , entretanto, a rapidez com que a temperatura tende para a temperatura da corrente livre depende fortemente do valor do Número de Prandtl (Pr).

0 aspecto mais relevante associado à Equação 08 é que a solução da camada-limite térmica é fortemente dependente da solução da camada-limite hidrodinâmica, uma vez que a função $f$ aparece na equação. Quão mais precisa a solução da camada-limite hidrodinâmica, mais precisa a solução da camada-limite térmica.

\section{OBJETIVOS}

Ao escrever o presente trabalho, tem-se em mente os estudantes que se iniciam em Mecânica dos Fluidos e se deparam com o conceito de camada-limite. Apesar de extensa literatura que trata do problema de camada-limite em placa plana, não existe, em um único texto, uma síntese clara dos procedimentos e conceitos envolvidos, e uma apresentação de todos os aspectos relacionados a ele (PASSOS, 2010). Pretende-se, então, em um único local, revisar os conceitos e apresentar algumas questões ainda indefinidas. Entende-se que uma introdução adequada do problema possa vir a ser de muita ajuda aos novatos que se aventuram aos problemas de camada-limite, uma vez que ainda estão sendo publicados inúmeros trabalhos, com generalizações do problema de Blasius. Um caso clássico de generalização, e extensamente referenciado na literatura, é o denominado Problema de Falkner-Skan, solucionado em 1932, onde a superfície encontra-se inclinada, com ângulo diferente de zero (EVANS, 1968). Além disso, conforme aponta Schlicting (1968), a solução de Blasius representa a solução básica para escoamento viscoso ao longo de uma superfície lisa.

Não é objetivo principal apresentar todos os passos na obtenção das equações básicas de camadalimite [Equações 06 e 08], mas sim discutir os métodos e os procedimentos necessários para obtenção da solução, e apresentar resultados relacionados com os mesmos, uma vez que existe vasta literatura na qual se demonstram esses passos (EVANS, 1968; KAYS AND CRAWFORD, 1983; SHAMES, 1973).

O poder do Método da Similaridade reside no fato de se ter uma equação ordinária a ser resolvida, ao invés de um sistema de equações parciais e, além disso, o procedimento e a solução envolvendo série ou Runge Kutta, se aproximam muito mais de um método analítico exato do que de um método numérico propriamente dito, de diferença finita, por exemplo.

Não se limita, aqui, na solução do Problema de Blasius, mas estende-se a análise para o problema da solução da Equação da Energia, ou seja, na obtenção da camada-limite térmica, onde pode-se obter o perfil de temperatura e o gradiente de temperatura, ao longo da camada-limite. 
Apresenta-se soluções para regime turbulento, em comparação ao regime laminar, tanto para perfil de velocidade quanto para perfil de temperatura. Nesse último caso, regime turbulento, usa-se o conceito de solução integral, denominado na literatura por Método de Von Karmann (KAYS AND CRAWFOR, 1983; FOX, MACDONALD, 1998). Além disso, usamos perfis de velocidade já consagrados na literatura, tais como Perfil de Expoente 1/7 e Perfil Universal (logaritmo) (KAYS; CRAWFORD, 1983; SCHLICHTING, 1968; DUTTON, 1959; DEEN, 1998).

\section{METODOLOGIA DE SOLUÇÃO}

Inicialmente, resolve-se o problema de condição de contorno definido pelas Equações 06 , seguindo os passos de Blasius, ou seja, usando uma expansão em série de potência. 0 método de aproximação utilizado, nesse caso, foi o Método da Bissecção. Esse tipo de procedimento não possibilita uma solução com a precisão necessária para os tempos atuais, mas permite uma solução aproximada mais adequada para a condição da derivada segunda na parede [f"(0)].

A partir da solução em série, descrita acima, utiliza-se o valor da derivada segunda na parede, como primeira aproximação, para solução da rotina de Runge Kutta de $4^{\text {a }}$ Ordem (BUTCHER, 2016). 0 método de aproximação utilizado foi o Método de Newton-Raphson, que permite maior precisão e é mais eficiente que o Método da Bissecção. Como, nesse caso, o valor de $f^{\prime}(\eta)$ para $\eta$ tende para 0 limite, tem-se melhor controle na escolha adequada desse valor. Os resultados obtidos demonstram a adequação do procedimento.

Com a solução esperada para f, f' e f" do problema hidrodinâmico, tem-se, agora, como atacar adequadamente o problema relacionado com o campo de temperatura. Para solução do problema em questão, integra-se diretamente a Equação 08. De fato, pode-se escrever:

$$
\begin{array}{ll} 
& \frac{d}{d \eta}\left(\theta^{\prime}\right)+\operatorname{Pr} . f \cdot \theta^{\prime}=0 \\
\rightarrow & \frac{1}{\theta^{\prime}} \frac{d}{d \eta}\left(\theta^{\prime}\right)+\operatorname{Pr} . f=0 \\
\rightarrow & \frac{d}{d \eta}\left(\ln \theta^{\prime}\right)=-P r . f \\
\rightarrow & d\left(\ln \theta^{\prime}\right)=-P r . f \cdot d \eta \\
\rightarrow & \int d\left(\ln \theta^{\prime}\right)=-\int \operatorname{Pr} . f \cdot d \eta
\end{array}
$$

Finalmente,

$$
\theta^{\prime}=\theta_{0}^{\prime} \cdot \exp \left[-P r \cdot \int_{0}^{\eta} f \cdot d \eta\right]
$$

Integrando novamente:

$$
\theta=\theta_{0}^{\prime} \cdot \int_{0}^{\eta} \exp \left[-\operatorname{Pr} \cdot \int_{0}^{\eta} f \cdot d \eta\right] d \eta
$$


onde $\theta_{0}^{\prime}$ é o valor da derivada da temperatura na superfície da placa. Logo, como

$$
\theta \rightarrow 1, \quad \eta \rightarrow \infty \quad \theta_{0}^{\prime}=\frac{1}{\int_{0}^{\infty} \exp \left[-P r \cdot \int_{0}^{\eta} f \cdot d \eta\right] d \eta}
$$

Observe que a obtenção da temperatura, e de sua derivada depende da determinação precisa de . Entretanto, quando se utiliza métodos numéricos usuais para a integração, a Equação 11 não possibilita uma adequada precisão para a determinação desse valor, quando comparada com resultados experimentais.

Evans (1968) preconiza a utilização do seguinte procedimento para obtenção da derivada da temperatura na superfície, com excelente precisão para .

$$
\left(\frac{d \theta}{d \eta}\right)_{0}=\frac{3}{E}\left(\frac{P r \cdot f_{0}^{\prime \prime}}{3 !}\right)^{1 / 3}
$$

onde

$$
E=2.6789385+\sum_{q=0}^{\infty} \frac{a_{q}}{\operatorname{Pr}^{q / 3}}
$$

Os valores de a para $q=1,2,4,5,7,8$ são iguais a zero.

$$
a_{3}=0.0595320 ; a_{6}=-0.00661466 ; a_{9}=0.000717145
$$

Com nove valores na série, e com a solução precisa da derivada segunda do perfil de velocidade [f"(0)], tem-se valores razoavelmente satisfatórios para o perfil de temperatura e sua derivada, para número de Prandtl $(\mathrm{Pr})$ acima ou igual a 0,6. Evans (1968) apresenta método de aproximação para Prandtl inferior a 0.6 , mas não é objetivo principal, como já salientado, discutir esse procedimento em detalhe neste trabalho.

O valor do coeficiente de fricção em coordenadas similares, em função do número de Reynolds, pode ser obtido por:

$$
\frac{C_{f}}{2}=\frac{f_{0}^{\prime \prime}}{\sqrt{2 R e_{x}}}
$$

O número de Nusselt, em coordenadas similares, em função do número de Reynolds, pode ser obtido por:

$$
N u_{x}=\left(\frac{d \theta}{d \eta}\right)_{0} \sqrt{\frac{R e_{x}}{2}}
$$

onde 


$$
R e_{x}=\frac{U . x}{\vartheta}
$$

O número de Stanton é obtido em função do número de Nusselt:

$$
S t_{x}=\frac{N u_{x}}{\operatorname{Pr} \cdot R e_{x}}
$$

$$
S t_{x}=\frac{C f / 2}{\sqrt{\frac{c f}{2}}(13.2 P r-10.16)+0.9}
$$

Salienta-se que essa última equação se aplica para regime turbulento (KAYS; CRAWFORD, 1983).

Para que se possa ter uma ideia da ordem de grandeza das propriedades em regime turbulento e para que se possa efetuar algumas comparações, torna-se imprescindível, aqui, apresentar alguns detalhes do Método Integral da Quantidade de Movimento. 0 poder do Método Integral reside no fato de que ele não é fortemente dependente do perfil de velocidade na camada-limite.

A equação integral da quantidade de movimento possibilita determinar, com razoável precisão, a espessura da camada-limite laminar ou turbulenta. Antes de se apresentar a expressão da equação integral, considere a seguinte "Lei de Potência", dada pela expressão (KAYS; CRAWFORD, 1983):

$$
u^{+}=8.75 y^{+1 / 7}
$$

onde

$$
u^{+}=\frac{U}{\sqrt{\frac{\tau_{w}}{\rho}}} \quad y^{+}=\delta \frac{\sqrt{\frac{\tau_{w}}{\rho}}}{\vartheta}
$$

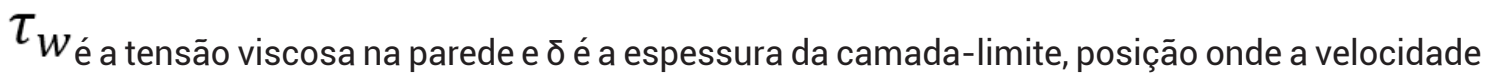
local é U.

Considera-se que a Equação 20, como primeira aproximação, é válida ao longo de toda camadalimite, ressaltando que foi deduzida para situações onde o gradiente de pressão é nulo. Assim,

$$
\begin{aligned}
& \frac{U}{\sqrt{\frac{\tau_{w}}{\rho}}}=8.75\left(\delta \frac{\sqrt{\frac{\tau_{w}}{\rho}}}{\vartheta}\right)^{1 / 7} \\
& \tau_{w}=0.0255 \rho U^{2}\left(\frac{\delta U}{\vartheta}\right)^{-1 / 4}
\end{aligned}
$$

Para placa plana, com gradiente de pressão nulo, a equação integral de quantidade de movimento reduz-se a: 


$$
\tau_{w}=\rho U^{2} \frac{d}{d x} \int_{0}^{\delta} \frac{u}{U}\left(1-\frac{u}{U}\right) d y
$$

Após aplicação do processo de integração, tem-se que:

$$
\frac{\tau_{w}}{\rho U^{2}}=\frac{C f}{2}=\frac{d \delta_{2}}{d x}+(H+2) \frac{\delta_{2}}{U} \frac{d U}{d x}
$$

$\mathrm{H}$ é denominado fator de forma, relação entre a espessura de deslocamento e a espessura de quantidade de movimento:

$$
H=\frac{\delta_{1}}{\delta_{2}}=\frac{\int_{0}^{\infty}\left(1-\frac{u \rho}{U \rho_{\infty}}\right) d y}{\int_{0}^{\infty} \frac{\rho u}{U \rho_{\infty}}\left(1-\frac{u}{U}\right) d y}
$$

Considerando que $\mathrm{H}=1,3$ (FOX; MACDONALD, 1998) para gradiente de pressão nulo, o coeficiente de friç̧ão para a Lei de Potência é obtido por:

$$
\frac{C f}{2}=\frac{0.0287}{R e_{x}{ }^{1 / 5}}
$$

Schultz-Grunow (1941), desenvolveu uma equação empírica, a partir de dados experimentais, para o coeficiente de fricção:

$$
\frac{C f}{2}=0.185\left(\log _{10} R e_{x}\right)^{-2.584}
$$

\section{RESULTADOS}

Os resultados clássicos da literatura, associados à solução de Blasius são as funções $f$, $f^{\prime}$ e f". Abaixo, no Gráfico 01 e Tabela 01, são apresentados os resultados para essas funções, após aproximação em série e, finalmente, aplicação de rotina Runge Kutta de $4^{\mathrm{a}}$ ordem. 
Tabela 01 - Placa Plana - Método de Runge Kutta.

\begin{tabular}{llll}
\hline \multicolumn{1}{c}{$\boldsymbol{\eta}$} & \multicolumn{1}{c}{$\mathbf{f}$} & \multicolumn{1}{c}{$\mathbf{f}^{\prime}$} & \multicolumn{1}{c}{$\mathbf{f}^{\prime \prime}$} \\
\hline 0 & 0 & 0 & 0,4696 \\
\hline 0,1198 & 0,00337 & 0,05626 & 0,46954 \\
\hline 0,3594 & 0,03032 & 0,16862 & 0,4679 \\
\hline 0,599 & 0,08411 & 0,28011 & 0,46177 \\
\hline 0,9584 & 0,21421 & 0,44248 & 0,43844 \\
\hline 1,198 & 0,33257 & 0,54443 & 0,41084 \\
\hline 1,3178 & 0,4007 & 0,59261 & 0,39321 \\
\hline 1,55741 & 0,55359 & 0,68193 & 0,35088 \\
\hline 1,79701 & 0,72659 & 0,76016 & 0,3011 \\
\hline 2,15641 & 1,01751 & 0,85391 & 0,22031 \\
\hline 2,39601 & 1,22793 & 0,90039 & 0,16839 \\
\hline 2,51581 & 1,33694 & 0,91911 & 0,14441 \\
\hline 2,99501 & 1,79073 & 0,96872 & 0,06832 \\
\hline 3,11481 & 1,90723 & 0,97607 & 0,05475 \\
\hline 3,71382 & 2,4987 & 0,99481 & 0,01464 \\
\hline 3,95342 & 2,7374 & 0,99743 & 0,00782 \\
\hline 4,19302 & 2,97657 & 0,99879 & 0,00394 \\
\hline 4,67222 & 3,45548 & 0,99977 & 0,00084 \\
\hline 4,91182 & 3,69504 & 0,99991 & 0,00036 \\
\hline 5,15142 & 3,93463 & 0,99997 & 0,00014 \\
\hline 5,27122 & 4,05443 & 0,99998 & $8,9 \mathrm{E}-05$ \\
\hline 5,51082 & 4,29403 & 0,99999 & $3,3 \mathrm{E}-05$ \\
\hline
\end{tabular}

Fonte: Dos autores, 2018.

Gráfico 1 - Funções f, f' e f" ao longo da camada-limite.

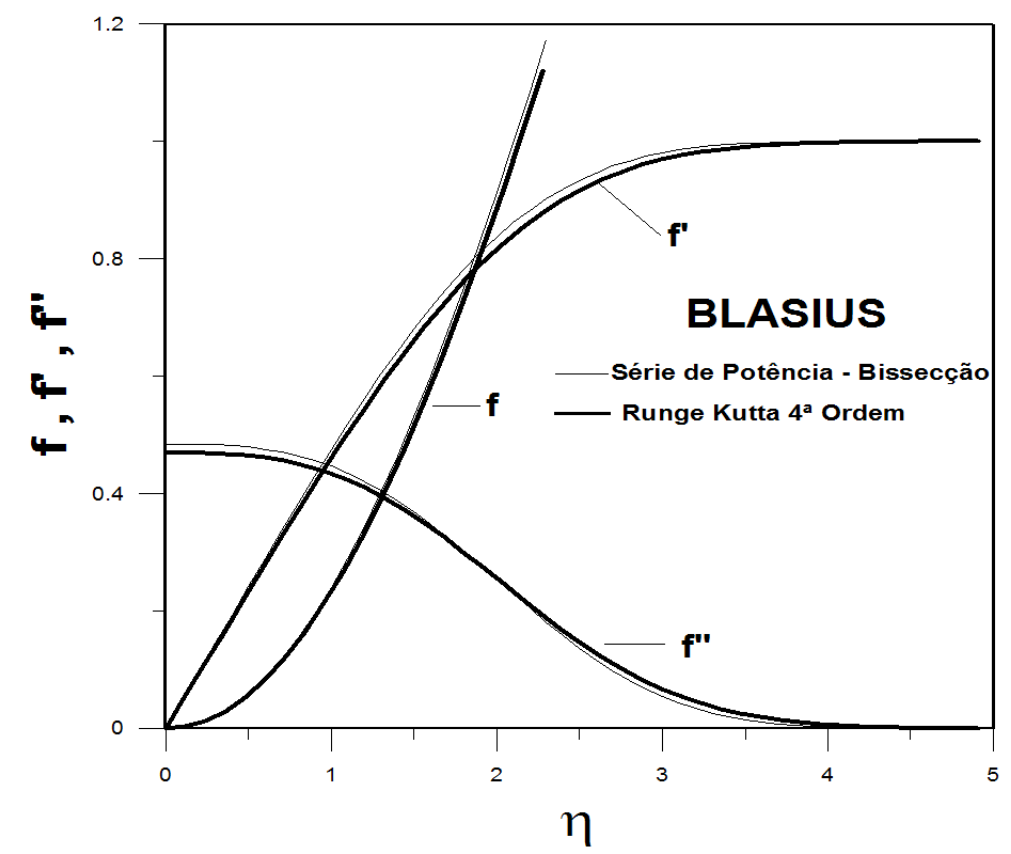

Fonte: Dos autores, 2018. 
Gráfico 2 - Coeficiente de fricção em função do número de Reynolds.

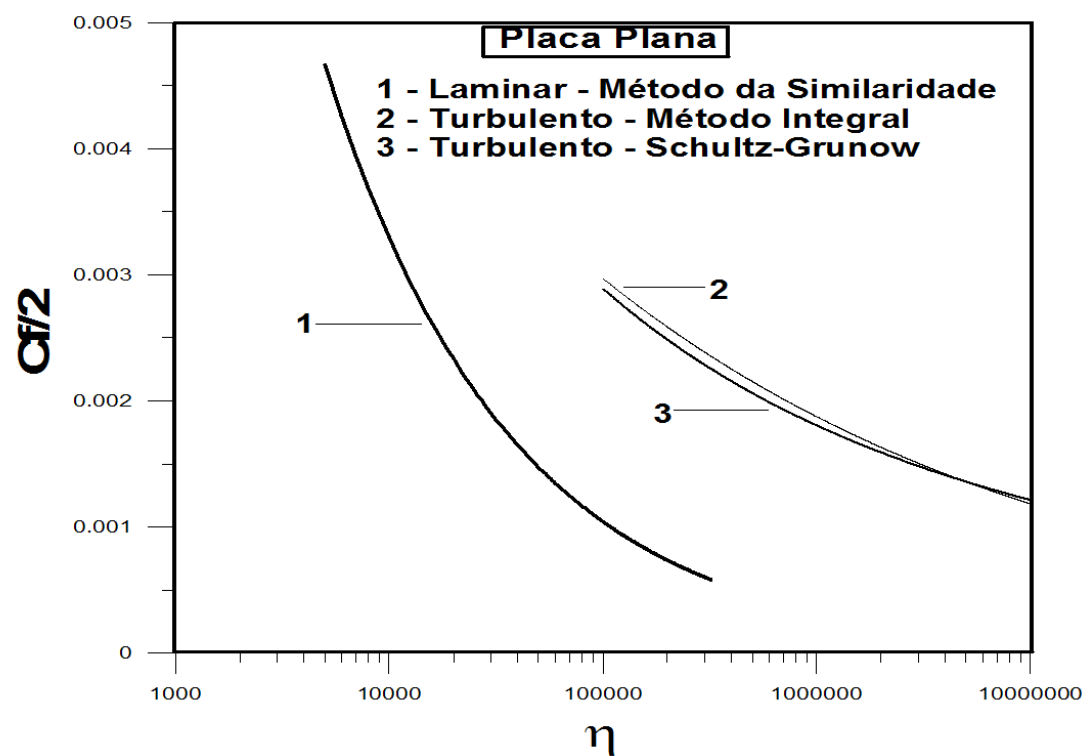

Fonte: Dos autores, 2018

No Gráfico 2, tem-se resultados para coeficiente de fricção em função do número de Reynolds, para regime laminar e turbulento.

Gráfico 3 - Temperatura adimensional na camada-limite em função do número de Pr.

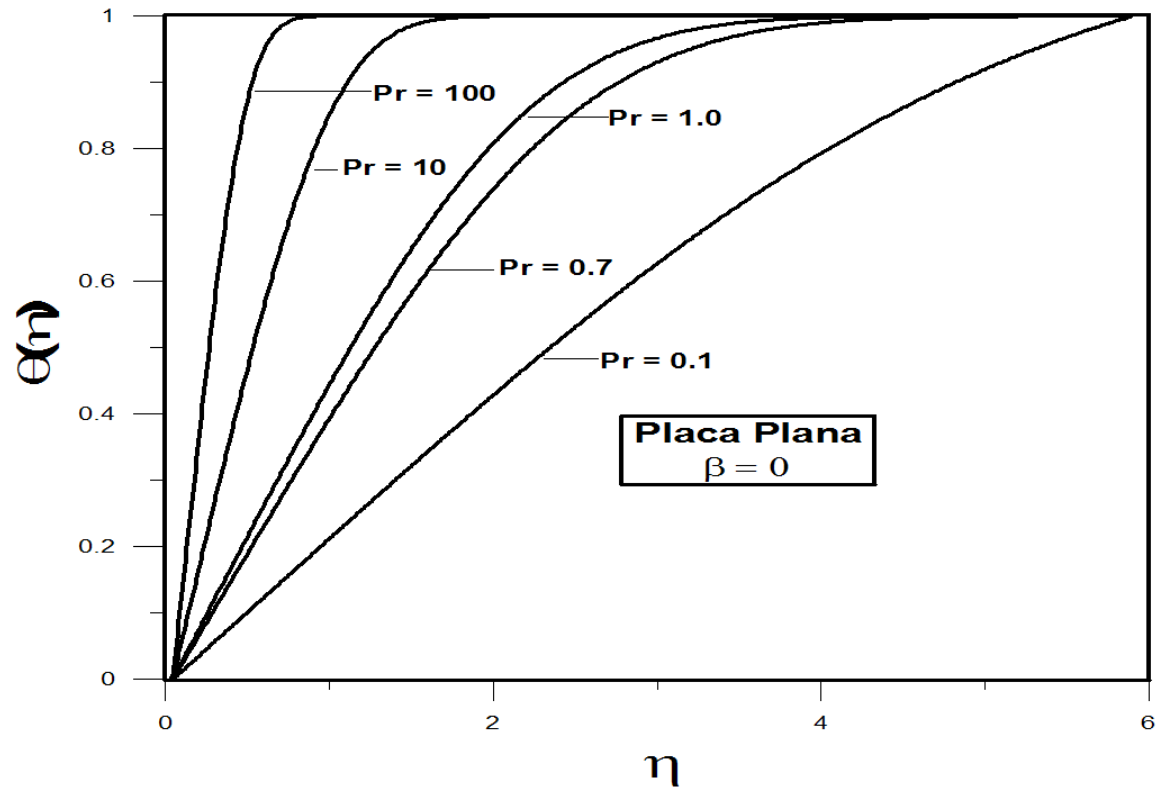

Fonte: Dos autores, 2018.

Na Gráfico 03, tem-se os resultados do perfil de temperatura adimensional em função do número de Prandtl. Observa-se, inicialmente que, para $\operatorname{Pr}=0.1$, a curva não apresenta um comportamento esperado, em função, como já salientado anteriormente, de que o procedimento preconizado é satisfatório para Pr>0.6. Além disso, como já esperado, uma vez que a espessura de condução diminui com a acréscimo do número de Prandtl, a espessura da camada-limite térmica é maior para Prandtl abaixo 
de 1.0, e diminui à medida que o número de Prandtl aumenta, uma vez que a difusividade de quantidade de movimento é superior à difusividade térmica para número de Prandtl acima da unidade.

Figura 4 - Gradiente de temperatura na superfície, em função do número de Prandtl.

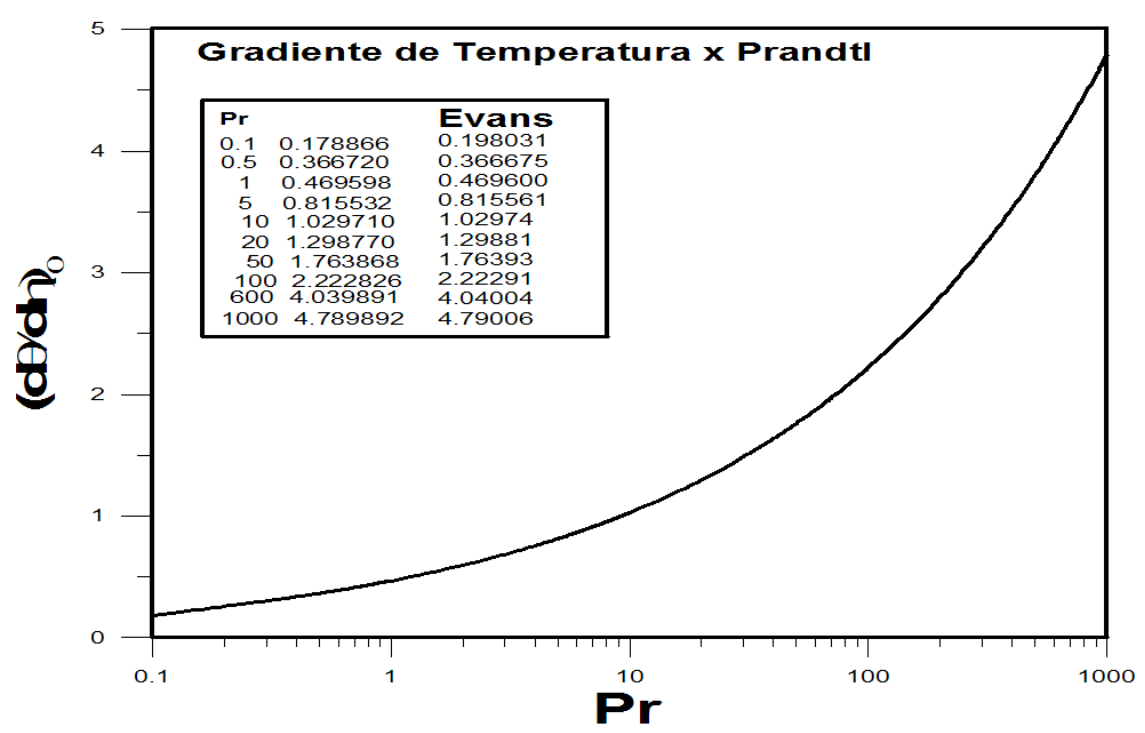

Fonte: Dos autores, 2018.

Os resultados acima, Gráfico 4, apresentam o gradiente de temperatura na superfície, em função do número de Prandtl. Esses resultados são de extrema importância para obtenção dos perfis de temperatura e gradiente de temperatura ao longo da camada-limite. A tabela em anexo ao gráfico demonstra o grau de definição dos resultados obtidos, em relação aos valores apresentados por Evans (1968).

Os resultados abaixo, Gráfico 5, apresentam o gradiente de temperatura ao longo da camada-limite, em função do número de Prandtl. Conforme observado anteriormente, Gráfico 03, a camada-limite térmica fica menos espessa à medida que o número de Prandtl cresce, como resultado do aumento na difusividade de quantidade de movimento. 
Gráfico 5 - Gradiente de temperatura na camada-limite, em função do número de Prandtl.

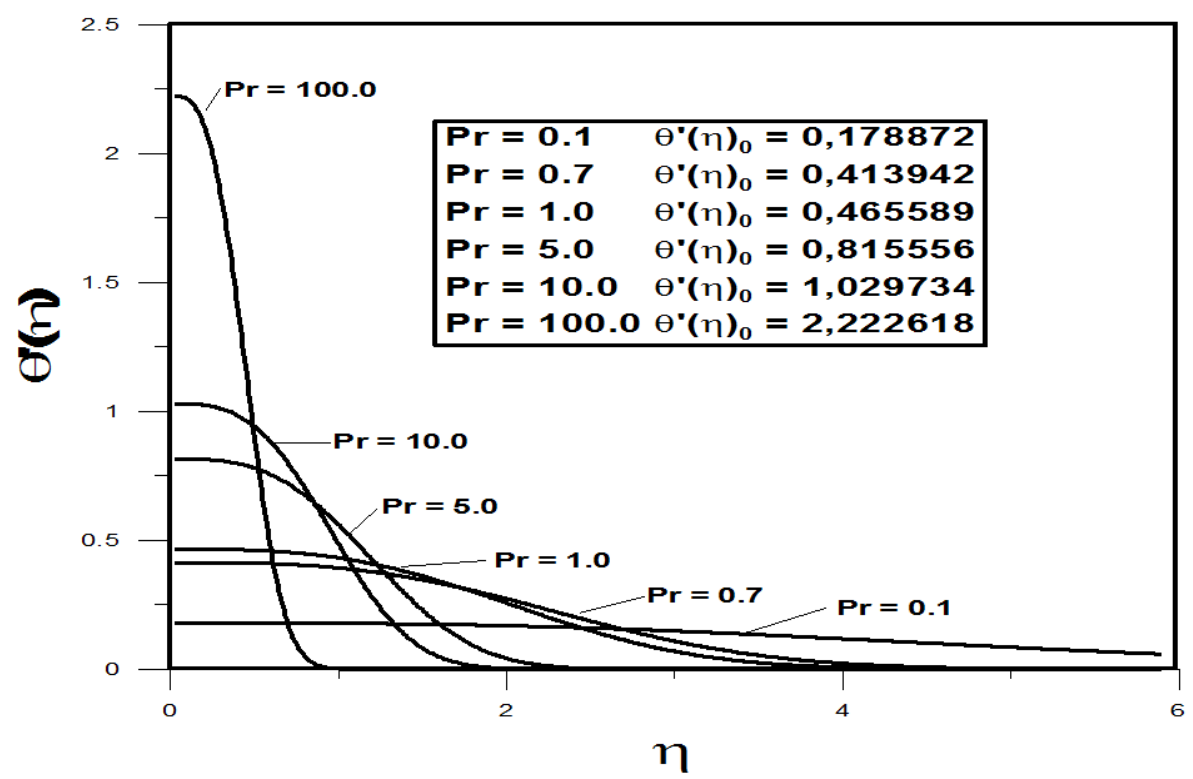

Fonte: Dos autores, 2018

Gráfico $6-\boldsymbol{N u} / \sqrt{\boldsymbol{R} \boldsymbol{e}_{\boldsymbol{L}}}$ versus X/L para camada-limite, regime laminar em placa plana.

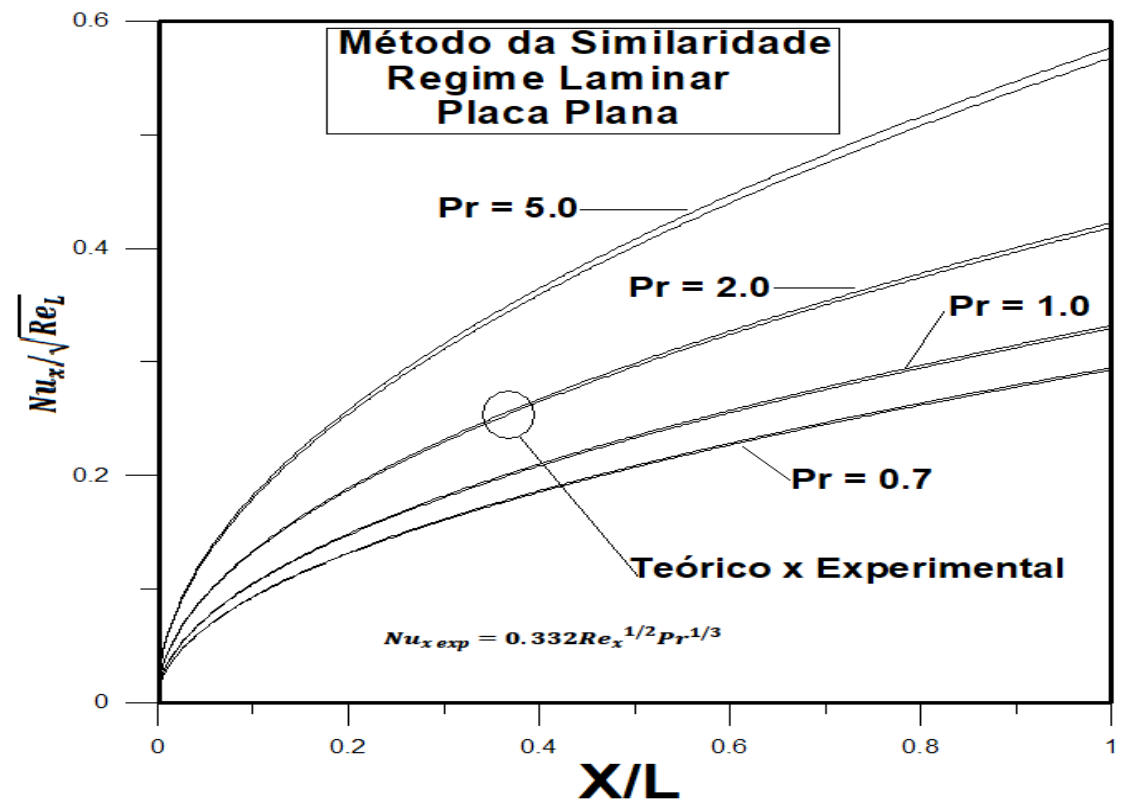

Fonte: Dos autores, 2018

A Gráfico 6 apresenta resultados para $\boldsymbol{N u} / \sqrt{\boldsymbol{R} \boldsymbol{e}_{\boldsymbol{L}}}$ versus $\mathrm{x} / \mathrm{L}$, em comparação com resultados clássicos para placa plana, onde $\mathrm{Re}_{L}$ é o número de Reynolds associado à um comprimento genérico $\mathrm{L}$ da placa, e x/L é o comprimento adimensional ao longo da placa. A comparação entre os resultados é satisfatória, dentro da faixa de Prandtl analisada. Em número de Prandtl relativamente baixo, os resultados obtidos pelo Método da Similaridade, implantado neste estudo, suplantam os resultados clássicos, e para Prandtl mais elevados ocorre o inverso e a diferença entre os resultados tendem a aumentar. 
Gráfico 7 - Número de Stanton para perfil laminar e turbulento em placa plana.

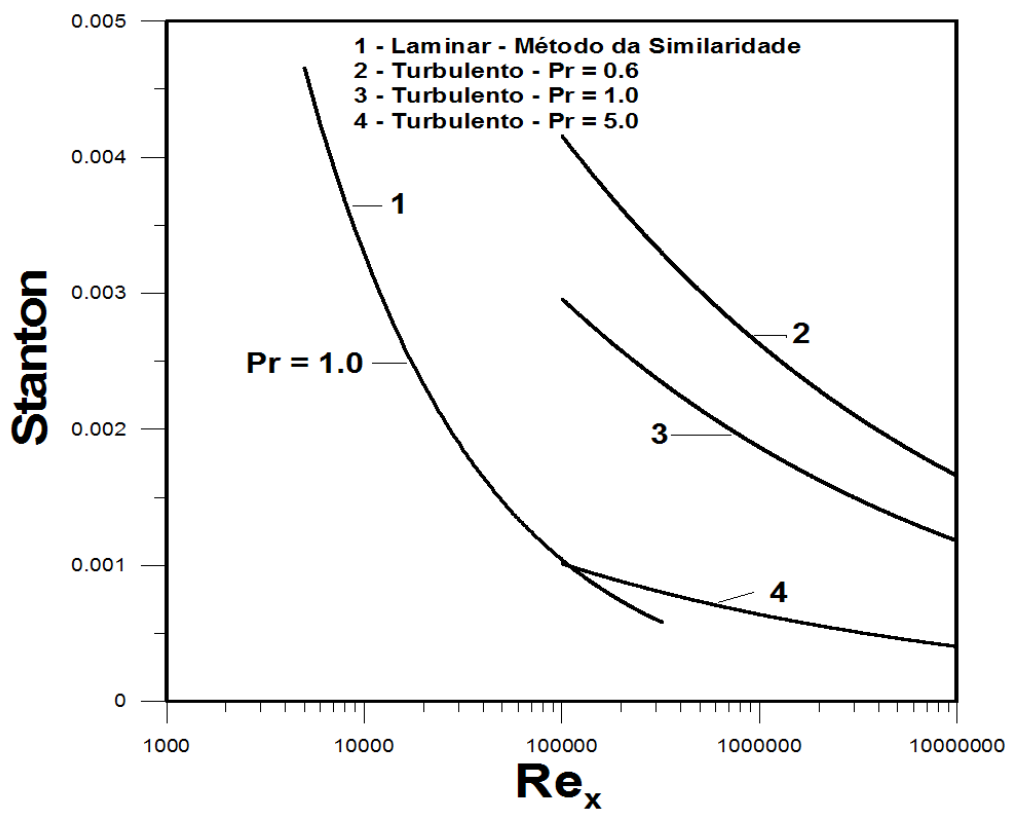

Fonte: Dos autores, 2018.

Os resultados da Gráfico 7 apresentam a variação do número de Stanton em função do número de Reynolds ao longo da placa. Eles demonstram que o número de Stanton decresce com o acréscimo do número de Prandtl, uma vez que é inversamente proporcional a este. Fato notável é que os resultados das Figuras 02 e 09 coincidem numericamente para número de Prandtl igual à unidade, em regime laminar, ou seja, $S t=\frac{C f}{2}$. Essa igualdade numérica ocorre, quando a difusividade de quantidade de movimento é igual à difusividade de energia na forma de calor, situação denominada por Analogia de Reynolds. De fato, conforme procedimento adotado neste trabalho, a diferença numérica entre os valores é insignificante, dentro da precisão estabelecida, o que pode ser constatado através da Tabela 02, abaixo.

Tabela 2 - Coeficiente de Fricção e número de Stanton, em função do número de Reynolds. Regime laminar, $\operatorname{Pr}=1.0$

\begin{tabular}{lll}
\hline $\mathbf{R e}_{x}$ & $\mathbf{C f} / \mathbf{2}$ & $\mathrm{St}$ \\
\hline 1000 & 0.0104109088964413 & 0.0104109087800903 \\
\hline 20000 & 0.00232794988633058 & 0.00232794987672862 \\
\hline 30000 & 0.00190076327744451 & 0.00190076328267115 \\
\hline 40000 & 0.00164610947239256 & 0.00164610948049692 \\
\hline 50000 & 0.00147232514336133 & 0.00147232512377387 \\
\hline 60000 & 0.00134404286541979 & 0.00134404286458664 \\
\hline 70000 & 0.00124434196341373 & 0.0012443419821466 \\
\hline 80000 & 0.00116397534100845 & 0.00116397534379746 \\
\hline 90000 & 0.00109740648758896 & 0.0010974064794141 \\
\hline 100000 & 0.00104109121498518 & 0.00104109119902988 \\
\hline
\end{tabular}

Fonte: Dos autores, 2018.

Os conceitos e procedimentos desenvolvidos para placa plana podem ser replicados de forma automática, por exemplo, para solução de camada-limite em superfícies inclinadas, como aquelas 
que ocorrem em uma falésia (PIRES, 2009; GARRATT, 1990; SILVA, 2014; STULL, 1998), problema de importância fundamental na área de Meteorologia. Além disso, como enfatizado por Evans, podem servir de base para soluções de camada-limite que não satisfazem o conceito de similaridade, e para problemas de camada-limite em geral. Spalding and Pun (1962) argumentam que a utilização do Método da Similaridade, e suas funções, como meio auxiliar, possibilitam maior precisão às soluções para camada-limite térmica. Para chegarem a tal conclusão, os autores analisaram dezesseis métodos distintos para solução de camada-limite em torno de um cilindro. De fato, o Método da Similaridade, apesar das Soluções Numéricas Diretas - DNS implementadas para solução das Equações de NavierStokes atualmente, continua sendo uma ferramenta utilizada para soluções de problemas associados à camada-limite, como se pode observar pela literatura apresentada nas referências.

\section{CONCLUSÃO}

Amplos resultados, numéricos e gráficos são apresentados e discutidos, com o objetivo de demonstrar o procedimento de solução de camada-limite em placa plana, hidrodinâmica e térmica, através do Método da Similaridade. Apesar de ser um assunto amplamente divulgado na literatura, com resultados parciais apresentados em livros didáticos de Mecânica dos Fluidos e Transmissão de Calor, uma revisão detalhada dos procedimentos utilizados para se chegar aos resultados fica a desejar (PASSOS, 2010).

O Método de Integração da Quantidade de Movimento, utilizado para solução aproximada de camada-limite turbulenta, foi apresentado com o intuito de ampliar a visão do estudante que se inicia em camada-limite, para efeito de comparações entre os dois tipos de regimes de escoamento. 0 número de Prandtl é considerado uniforme ao longo de toda a camada-limite, mas isso é uma aproximação, uma vez que há variação da difusividade turbulenta para Prandtl ao longo da camada-limite, ou seja, o número de Prandtl Turbulento varia na camada-limite (LI ET AL., 2015). Assumir um valor uniforme para $\operatorname{Pr}$ ao longo da camada-limite é uma hipótese razoável, se a diferença de temperatura, entre a temperatura da corrente livre e a temperatura da superfície não for significativa. 


\section{REFERÊNCIAS}

ABBASI, M. et al. Analytic Solution of Hydrodynamic and Thermal Boundary Layer over a Flat Plate in a Uniform Stream of a Fluid With Convective Surface Boundary Condition. Indian J.Sci.Res.1(2): 241-247, 2014.

BHATTACHARYYA, K. et al. Exact Solution for Thermal Boundary Layer in Casson Fluid Flow over a Permeable Shrinking Sheet with Variable Wall Temperature and Thermal Radiation. Alexandria Engineering Journal 55, 1703-1712, 2016.

BOGNAR, G.; HRICZÓ. Similarity Solutions a Thermal Boundary Layer Models of a non-Newtonian Fluid with a Convective Surface Boundary Condition. Acta Polytechnica Hungarica, Vol 8, No. 6, 2011.

BUTCHER, J. C. Numerical Methods for Ordinary Equations. Wiley, 2016.

CASTILHO, L. Similarity Analyses of Turbulent Boundary Layer. A Dissertation Submitted in a Partial Requirement for the Degree of Doctor of Philosophy, State University of Buffalo, 1997.

DEEN, W. M. Analysis of Transport Phenomena. Oxford University Press, 1998.

DUTTON, R. A. The Velocity Distribution in a Turbulent Boundary Layer a Flat Plate. A.R.C Technical Report, Aeronautical Research Council, London, 1959.

EVANS, Harry L. Laminar Boundary-Layer Theory. ADDISON-WESLEY PUBLISHING COMPANY, 1968.

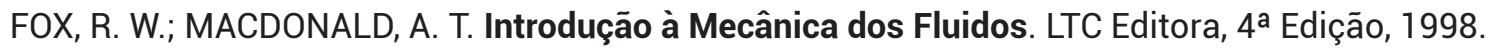

GARRATT, J. R. The Internal Boundary Layer - A Review. Boundary-Layer Meteorology 50: 171-203, 1990.

HAGER, W. H. Blasius: A Life in Research and Education. Experiments in Fluids, 34, 566-571, 2003.

KAYS, W. M.; CRAWFORD, M. E. Convective Heat and Mass Transfer. Tata McGraw-Hill Publising Co. Ltd, New Delhi, 1983.

LI, D. et al. Revisiting the Turbulent Prandtl Number in a Idealized Atmosphere Surface Layer. Journal of the Atmospheric Science, Vo. 72, 2394-2410, 2015.

MARTIN, M. J. et al. Momentum and Heat Transfer in a Laminar Boundary Layer with Slip Flow. Journal of Thermophysics and Heat Transfer, Vol. 20, No. 4, October-December, 2006.

MYERS, T. G. An Approximate Solution Method for Boundary Layer Flow of A Power Law Fluid Over a Flate Plate. International Journal of Het and Mass Transfer, 53, 2337-2346, 2010.

ODERINU, R. A. Shooting Method via Taylor Series for Solving Two Point Boundary Value Problem on an Infinite Interval. Gen. Math. Notes, Vol. 24, No. 1, September, pp. 74-83, 2014.

PASSOS, J. C. 0 Conceito de Camada Limite: Uma Revisão Crítica de Livros Textos de Transferência de Calor. VI Congresso Nacional de Engenharia Mecânica, 18 a 21 de agosto, Campina Grande, Paraíba-Brasil, 2010. 
PIRES, L. B. Marinho Estudo da Camada Limite Interna Desenvolvida em Falésias com Aplicação para o Centro de Lançamento de Alcântara. Tese de Doutorado do Curso de Pós-Graduação em Meteorologia, Inpe, São José dos Campos, 2009.

PUTTKAMMER, P.P. Boundary Layer over a Flat Plate. BSc Report, University of Twente, Enschede, June 2013.

RAHMAN, M. M. "Locally Similar Solutions for a Hydromagnetic and Thermal Slip Flow Boundary Layers over a Flate Plate with Variable Fluid Properties and Convective Surface Boundary Condition". Meccanica, 46:1127-1143. DOI 10.1007/s11012-010-9372-2, 2011.

SCHLICHTING, H. Boundary-Layer Theory. McGraw-Hill Book Company, New York, 1968.

SCHULTZ-GRUNOW, F. NA TM-986 (now NASA), Washington, 1941.

SHAMES, I. H. Mecânica dos Fluidos: Análise de Escoamentos. Editora Edgard Blucher Ltda, Vol. 2. Tradução: Mauro O. C. Amorelli, 1973.

SILVA, A. F. G.; FISCH, G. Avaliação do Modelo WRF para a Previsão do Perfil do Vento no Centro de Lançamento de Alcântara. Revista Brasileira de Meteorologia, V. 29, n.2, 259-270, 2014.

SPALDING, D. B.; Pun, W. M. A Review of Methods for Predicting Heat-Transfer Coefficients for Laminar Uniform-Property Boundary Layers Flows. Int. J. Heat Mass Transfer, Vol. 5, pp. 239-249, Pergamon Press 1962.

STEMMER, C. "Boundary Layer Theory. Falkner-Scan Solution (Flat-Plate Boundary Layer with Pressure Gradients)". Lehrstuhf für Aerodynamik, Tecnische Universität Münchem, Boundary Layer Theory, GIST 2010.

STULL, R. B. An Introduction on Boundary Layer Meteorology. Atmospheric Sciences Library, Kluwer Academic Publishers, London, 1988. 